\title{
Apical but not sub-apical hyphal compartments are self-sustaining in growth
}

\author{
Martin Tegelaar · George P. A. van der Lans • Han A. B. Wösten
}

Received: 22 October 2019/Accepted: 2 January 2020/Published online: 9 January 2020

(C) The Author(s) 2020

\begin{abstract}
It was recently demonstrated that apical compartments of Aspergillus niger hyphae are selfsustaining in growth. This was shown by assessing the growth rate of individual hyphae before and after dissection of the second compartment. Using the same methodology, it is here demonstrated that single apical compartments of the septate fungi Penicillium chrysogenum and Schizophyllum commune as well as the $500-\mu \mathrm{m}$-apical region of the non-septate fungus Rhizopus stolonifer are also self-sustaining in growth. In contrast, single 2nd compartments (obtained by dissection of the first and third compartment) of the septate fungi or the region between 500 and $1000 \mu \mathrm{m}$ from tips of $R$. stolonifer were severely impacted in their growth rate. In addition, it is shown that existing or newly formed branches originating from the 2nd compartments function as a backup system for hyphal growth when the apical part of the hypha of the three studied fungi is damaged. Together, it is concluded that the apical compartments/zones of the studied fungi are self-sustaining in growth. In contrast, the subapical region is not self-sustaining but functions as a backup once the apical zone is damaged. This back up system is relevant in nature because the apices of
\end{abstract}

M. Tegelaar - G. P. A. van der Lans .

H. A. B. Wösten ( $\square)$

Microbiology, Department of Biology, Utrecht

University, Padualaan 8, $3584 \mathrm{CH}$ Utrecht,

The Netherlands

e-mail: h.a.b.wosten@uu.nl hyphae are the first to be exposed to (a)biotic stress conditions when entering an unexplored substrate.

Keywords Fungus $\cdot$ Hyphae $\cdot$ Compartment $\cdot$ Apical growth $\cdot$ Branching

\section{Introduction}

Filamentous fungi form mycelia that consist of a network of hyphae. These hyphae extend at their apices and branch sub-apically. Hyphae of mucoromycetes are generally non-compartmentalized, while those of ascomycetes and basidiomycetes are divided by septa. These septa have a $50-500 \mathrm{~nm}$ wide central pore flanked by invaginations of the cell wall lined with plasma membrane (Shatkin and Tatum 1959; Moore and McAlear 1962; Lew 2005; Steinberg et al. 2017). By plugging the pores, intercompartmental and interhyphal cytoplasmic mixing is interrupted (Bleichrodt et al. 2012, 2015a). Intact growing hyphae of Aspergillus oryzae (Bleichrodt et al. 2012), Aspergillus niger (Tegelaar et al. 2019), and Zymoseptoria tritici (2017b) plug their pores with Woronin bodies, while hyphae of the basidiomycete Schizophyllum commune close their pores with the septal pore cap (van Peer et al. 2010). Environmental conditions impact incidence of septal closure in S. commune (van Peer et al. 2009), A. oryzae and A. niger (Tegelaar et al. 
2019). Low glucose levels reduce plugging incidence in $S$. commune, while presence of antibiotics, heat shock, and hypertonic shock promote septal closure (van Peer et al. 2009). Plugging incidence of septa of A. oryzae is affected by heat treatment, low $\mathrm{pH}$ conditions, $\mathrm{C}$-starvation and $\mathrm{N}$-starvation, while that of A. niger is affected by exposure to heat, high $\mathrm{pH}$, and hypertonic conditions (Tegelaar et al. 2019). Incidence of septal closure in Aspergillus also increases in time. Half of the newly formed apical septa are open, while septa flanking the 10th compartment are always closed (Bleichrodt et al. 2012, 2015a). From this and the fact that septal closure abolishes bulk mixing of cytoplasm it was concluded that hyphal compartments transform from a unicellular to a multicellular system (Bleichrodt et al. 2015a). Yet, selective transport is still possible via transporters in the septal plasma membrane (Bleichrodt et al. 2015b).

The fact that apical compartments of Aspergillus hyphae form a unicellular system is in line with the concept of the peripheral growth zone. This zone defines the number of compartments that are needed for a hypha to maintain its growth rate (Trinci 1971). The peripheral growth zones of A. niger and Penicillium chrysogenum would consist of 11 and 13 intact compartments and $8660 \mu \mathrm{m}$ for the non-septate hyphae of Rhizopus stolonifer. Notably, it was recently shown that apical compartments of $A$. niger are selfsustaining (Tegelaar and Wösten 2017), thus reducing its peripheral growth zone to one compartment. This difference was explained by the fact that Trinci (1971) determined the growth rate of colonies before and after damaging the mycelium at different lengths from the outer part of the colony, while Tegelaar and Wösten (2017) assessed growth after dissection of individual hyphae. Using the latter methodology, it is here shown that single apical compartments of the ascomycete $P$. chrysogenum and the basidiomycete $S$. commune and the first $500 \mu \mathrm{m}$ of hyphae of $R$. stolonifer are also self-sustaining in growth. In contrast, single 2nd compartments (obtained by dissection of the first and third compartment) of septate fungi or the region between 500 and $1000 \mu \mathrm{m}$ from the tip of $R$. stolonifer were severely impacted in their growth rate.

\section{Materials and methods}

Strains and growth conditions

Strains N402 of A. niger (Bos et al. 1988), $P$. chrysogenum Wisconsin 54-1255 (Stauffer 1961), $R$. stolonifer CBS 112376 and S. commune H4-8 (matA43 matB41; FGSC 9210 (Ohm et al. 2010) were used in this study. The former two fungi were grown at $30{ }^{\circ} \mathrm{C}$ in water-saturated air in the dark, while the latter two fungi were grown at $25^{\circ} \mathrm{C}$ and $50 \%$ humidity in 750 lx white light (C65 $100 \mathrm{~mA}$ 5730, NS12 spectrum, Valoya, Helsinki, Finland). $R$. stolonifer plates were sealed with cellophane. Spores of A. niger and $P$. chrysogenum were harvested in $10 \mathrm{ml} 0.9 \% \mathrm{NaCl}(\mathrm{w} / \mathrm{v}), 0.05 \%$ (v/v) Tween-20 from 7-day-old cultures grown in $9 \mathrm{~cm}$ Petri dishes on complete medium (CM). This medium consisted of minimal medium (MM; $0.6 \% \mathrm{NaNO}_{3}, 0.15 \% \mathrm{KH}_{2}$

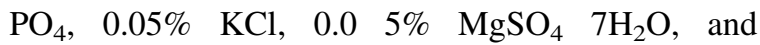
$0.2 \mathrm{ml} \mathrm{l}^{-1}$ Vishniac solution (Vishniac and Santer 1957), $\mathrm{pH}$ 6.0) supplemented with $0.5 \%$ yeast extract, $0.2 \%$ casamino acids, $25 \mathrm{mM}$ maltose and $1.5 \%$ agarose. Spores of $R$. stolonifer were obtained as described above, but harvested after 3 days. S. commune was grown on polycarbonate membranes (Whatman Cyclopore $^{\mathrm{TM}}, 76 \mathrm{~mm}$ diameter $0.1 \mu \mathrm{m}$ pore size, Osmonics; GE Water Technologies) placed on S. commune minimal medium (SCMM; Dons et al. 1979) solidified with $1.5 \%$ agarose. After 3 days the $S$. commune mycelium was macerated in $50 \mathrm{ml}$ SCMM for $1 \mathrm{~min}$ at high speed with a Waring Blender. The homogenated mycelium was transferred to a $50 \mathrm{ml}$ Greiner tube and was incubated for $24 \mathrm{~h}$ at $25{ }^{\circ} \mathrm{C}$ in the dark. Spores and micro-colonies obtained in these ways were used to inoculate glass bottom dishes (Cellview $^{\mathrm{TM}}$ cell culture dishes, PS, 35/10 mm, Greiner Bio-One, Frickenhausen, Germany). To this end, the dishes and medium were pre-warmed to $60{ }^{\circ} \mathrm{C}$. $0.5 \mu \mathrm{L}$ spore solution (50,000 spores), or one $S$. commune micro-colony, was placed on a glass coverslip (18 $\mathrm{mm}$ in diameter and $0.16-0.19 \mathrm{~mm}$ thick) and left to dry. Spores were allowed to dry completely, while micro-colonies were allowed to dry until no more medium was visible around the microcolony. $30 \mu \mathrm{L}$ pre-warmed MMA (MM supplemented with $25 \mathrm{mM}$ maltose and $1 \%$ agarose) or SCMMA (SCMM with $1 \%$ agarose), was added to the middle of the glass bottom dish, and immediately brought in 
contact with coverslip-adhered A. niger, P. chrysogenum and $R$. stolonifer spores or $S$. commune microcolonies, respectively. After the layer of medium had solidified, $2 \mathrm{ml}$ of corresponding liquid medium (i.e. (SC)MM supplemented with $25 \mathrm{mM}$ maltose) was pipetted on top of the coverslip. Laser dissection was performed after $48 \mathrm{~h}$ of growth.

\section{Microscopy}

Hyphae were dissected using a PALM Microbeam system (laser power $35 \%$, focus $57 \%$ ) linked to an Observer.Z1 inverted microscope (Carl Zeiss AG, Oberkochen, Germany) and a CCD color camera (AxioCam ICc 1, Carl Zeiss AG, Oberkochen, Germany). Growth rate of hyphae and branches was recorded every $5 \mathrm{~min}$ during a $15 \mathrm{~min}$ period prior to dissection. In addition, it was assessed whether septal pores, if present, were open or closed at the moment of dissection. Septa were classified as open when cytosol was leaking through the septal pore upon dissection (Bleichrodt et al. 2012). Hyphal growth after dissection was recorded every $5 \mathrm{~min}$ for $45 \mathrm{~min}$ for $P$. chrysogenum and $S$. commune hyphae cut in the 2 nd compartment. Growth from hyphae cut in the apex or in both the apex and 3rd compartment in A. niger and $P$. chrysogenum was recorded every 30 min during a $4 \mathrm{~h}$ period after dissection, with non-dissected hyphae serving as control. For $R$. stolonifer, growth was recorded every hour during a $4 \mathrm{~h}$ period after dissection, with non-dissected hyphae serving as control. Single 2nd compartments of S. commune were followed for $>7 \mathrm{~h}$ using the PALM RoboSoftware time-lapse function. Width and length of compartment pre- and post-dissection were also recorded, as well as translocation $(\mu \mathrm{m})$ of vacuoles after cutting. From these parameters the post-dissection volume of the apical compartment was calculated (Tegelaar and Wösten 2017).

\section{Statistics}

Experiments were performed using at least biological triplicates (i.e. independent cultures grown at different days), each with at least 7 technical replicates (i.e. repeated measures within a biological replicate). Single 2nd compartments of $S$. commune that were followed using time-lapse had 11 biological replicates and no technical replicates. $T$ tests or Mann-Whitney
$\mathrm{U}$ tests were carried out to determine differences in growth rate between treatment and control apical compartments. Differences in hyphal growth or volume before and after cutting were determined using paired sample t-tests, followed by correlation and regression analyses. Analysis of bimodality was performed as described (Vinck et al. 2011).

\section{Results}

Closure of a septum, or the presence of a septum at all, is not a prerequisite for continued growth after sub-apical hyphal damage

Growth rate of hyphae of $P$. chrysogenum, $S$. commune and $R$. stolonifer was $79 \pm 4,173 \pm 16$ and $102 \pm 20 \mu \mathrm{m} \mathrm{h}^{-1}$, respectively (for growth conditions see "Materials and methods" section) (Table 1). Mean hyphal growth rate was reduced by 8,4 , and $71 \%$ after dissection within the second compartment of $P$. chrysogenum and S. commune or $500 \mu \mathrm{m}$ from the apex in the case of $R$. stolonifer (called pseudo-apical compartment from now on), respectively (Table 1 ). This mean hyphal growth rate was calculated including the 9,4 , and $40 \%$ of the (pseudo-)apical compartments of $P$. chrysogenum, $S$. commune and $R$. stolonifer that stopped growing after dissection, respectively. Growth rate of single apical compartments of $P$. chrysogenum and $S$. commune was not affected when only hyphae were taken into account that remained growing after cutting. Notably, even apical hyphal fragments of the coenocytic $R$. stolonifer that continued growing did not show a significant reduction in extension (Table 1). The apical septum of all dissected S. commune hyphae was open before cutting, while $21 \%$ of the $P$. chrysogenum apical septa were closed. More specifically, 29 and $17 \%$ of the apical septa were closed of the $P$. chrysogenum apical compartments that stopped and continued growing after dissection, respectively. Together, these findings show that closure of a septum, or the presence of a septum at all, is not a prerequisite for continued growth after dissection. Yet, the presence of a septum contributes to the survival of hyphae after injury.

On average, the (pseudo-)apical compartments that halted their growth after dissection lost 11, 51 and $69 \%$ of their cytoplasm in the case of $P$. chrysogenum, 
Table 1 Length, width, and growth rate of the first compartment of $P$. chrysogenum and $S$. commune and the apical zone of $R$. stolonifer before and after laser dissection

\begin{tabular}{|c|c|c|c|c|c|c|}
\hline & \multirow{2}{*}{$\begin{array}{l}\text { Length } \\
(\mu \mathrm{m})\end{array}$} & \multirow{2}{*}{$\begin{array}{l}\text { Width } \\
(\mu \mathrm{m})\end{array}$} & \multicolumn{2}{|c|}{ Growth rate before dissection $\left(\mu \mathrm{m} \mathrm{h}^{-1}\right)$} & \multicolumn{2}{|c|}{ Growth rate after dissection $\left(\mu \mathrm{m} \mathrm{h}^{-1}\right)$} \\
\hline & & & All hyphae & $\begin{array}{l}\text { Growing hyphae } \\
\text { after dissection }\end{array}$ & All hyphae & $\begin{array}{l}\text { Growing hyphae } \\
\text { after dissection }\end{array}$ \\
\hline P. chrysogenum & $243 \pm 17$ & $4.5 \pm 0.05$ & $79 \pm 4$ & $79 \pm 4$ & $73 \pm 4$ & $80 \pm 4$ \\
\hline$N=353$ & $\mathrm{~N}=144$ & $\mathrm{~N}=174$ & $\mathrm{~N}=353$ & $\mathrm{~N}=320$ & $\mathrm{~N}=353$ & $\mathrm{~N}=320$ \\
\hline S. commune & $295 \pm 17$ & $4.1 \pm 0.16$ & $173 \pm 16$ & $174 \pm 15$ & $168 \pm 20$ & $175 \pm 20$ \\
\hline $\mathrm{N}=86$ & $\mathrm{~N}=86$ & $\mathrm{~N}=44$ & $\mathrm{~N}=85$ & $\mathrm{~N}=45$ & $\mathrm{~N}=47$ & $\mathrm{~N}=45$ \\
\hline R. stolonifer & 500 & $3.6 \pm 0.4$ & $102 \pm 20$ & $74 \pm 16$ & $30 \pm 10$ & $65 \pm 18$ \\
\hline $\mathrm{N}=47$ & $\mathrm{~N}=34$ & $\mathrm{~N}=24$ & $\mathrm{~N}=14$ & $\mathrm{~N}=7$ & $\mathrm{~N}=34$ & $\mathrm{~N}=18$ \\
\hline
\end{tabular}

Mean growth rate $( \pm 95 \%$ confidence interval) before and after dissection is indicated of all dissected hyphae and of those hyphae that continued growing after laser dissection

$S$. commune and $R$. stolonifer, respectively. In contrast, single apical fragments that continued their growth lost 1,13 and $27 \%$ of their cellular volume, respectively. These data show that growth of the apical fragments of the septate fungi $P$. chrysogenum and $S$. commune are more sensitive to loss of volume when compared to $R$. stolonifer.

Subapical compartments assume peripheral growth after dissection of the apex.

Hyphae of A. niger, $P$. chrysogenum, and $S$. commune were dissected in the middle of the apical compartment, while $R$. stolonifer was cut at the very tip. Growth from the 2nd compartment, or, in the case of $R$. stolonifer, sub-apical to the damaged apex was recorded after laser dissection. These compartments of A. niger, $P$. chrysogenum, $S$. commune and the 500- $\mu \mathrm{m}$-peripheral zone of $R$. stolonifer had formed lateral branches before dissection in 10, 18, 50 and $100 \%$ of the cases, respectively. The mean extension rate of these branches was $14.8 \pm 3.7,14.9 \pm 3.2$ and $148.2 \pm 20.6 \mu \mathrm{m} \mathrm{h}^{-1}$ for A. niger, P. chrysogenum and $S$. commune, respectively (Table 2). No growth was observed from previously formed branches in $R$. stolonifer. After laser dissection of the apex of the main hypha, previously formed lateral branches originating from the subapical zone continued their growth in 71, 78, 100 and $2 \%$ for A. niger, $P$. chrysogenum, S. commune and $R$. stolonifer and had a mean extension rate of $9.5 \pm 3.8,13.9 \pm 1.6$, $132 \pm 10.2$ and $43.6 \pm 23.8 \mu \mathrm{m} \mathrm{h}^{-1}$, respectively (Table 2). Thus, laser dissection of the apical zone of the studied fungi did not affect growth rate of branches that were previously formed from the 2 nd compartment.

After dissection of the apical compartment, newly formed branches appeared through the apical septum in 47, 57 and $10 \%$ of the hyphae of A. niger, $P$. chrysogenum and $S$. commune, respectively. These newly formed branches had a mean extension rate of $21.6 \pm 2.3, \quad 16.2 \pm 1.3$ and $56.3 \pm 54.9 \mu \mathrm{m} \mathrm{h}^{-1}$ (Table 2). In contrast, newly formed lateral branches originating from the 2 nd compartment had a mean extension rate of $16.1 \pm 2.1, \quad 31.2 \pm 5.4$ and $100 \pm 14.6 \mu \mathrm{m} \mathrm{h}^{-1}$. After apical injury, R. stolonifer hyphae placed a septum at a mean distance of $69 \pm 13 \mu \mathrm{m}$ from the apical wound whenever growth was resumed by branches that were newly formed under this new septum. These branches had a mean growth rate of $43.2 \pm 6.8 \mu \mathrm{m} \mathrm{h}^{-1}$ (Table 2). Together, data show that branches that had been formed before and after dissection have a similar growth rate after cutting the hyphae.

Growth from single (pseudo-)2nd compartments was recorded after dissection of hyphae both within the (pseudo-)apical and (pseudo-)3rd compartments. To this end, R. stolonifer was cut 500 and $1000 \mu \mathrm{m}$ from the apex. After dissection, 42, 94, 57 and $0 \%$ of previously formed lateral branches A. niger, $P$. chrysogenum, $S$. commune and $R$. stolonifer (pseudo)2nd compartments continued their growth. These branches had a mean extension rate of $14.1 \pm 12.4$, $27.3 \pm 8.9$, and $11.4 \pm 23.8 \mu \mathrm{m} \mathrm{h}^{-1}$ for A. niger, $P$. 


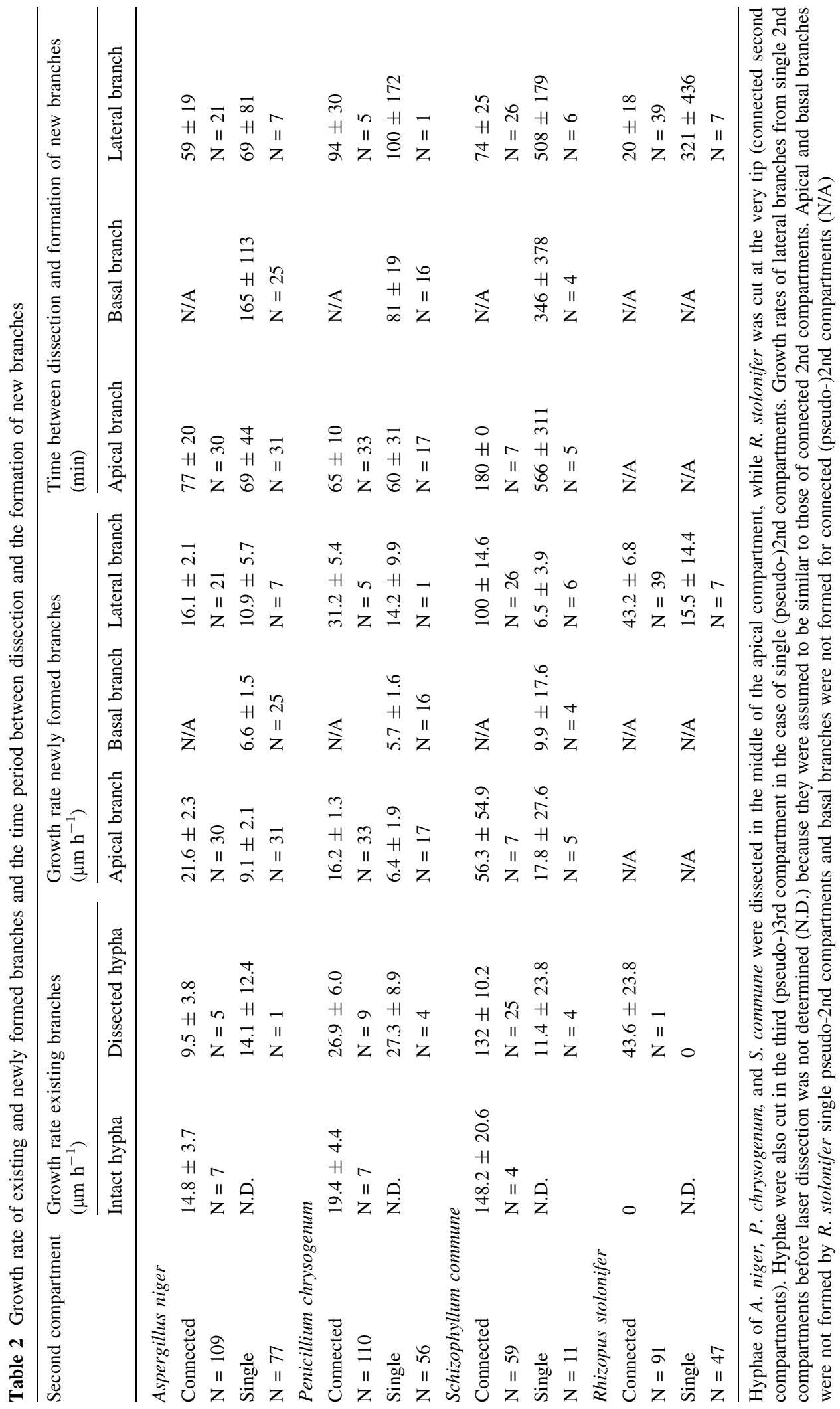


chrysogenum and S. commune, respectively (Table 2). Newly formed branches of single (pseudo-)2nd compartments grew through the apical or basal septum or were formed laterally. Lateral branches were formed in 8,2 and $50 \%$ of the cases in A. niger, $P$. chrysogenum and $S$. commune, respectively. Branches growing through the apical septum of the 2nd compartments were formed in 69,60 and $45 \%$ of the 2nd compartments of A. niger, $P$. chrysogenum and $S$. commune, respectively, while this was 43,37 and $36 \%$ for basal septa. In total, 79, 70 and $55 \%$ of single 2 nd compartments resumed growth by forming a branch through either apical, basal, or both septa for A. niger, $P$. chrysogenum and $S$. commune, respectively (Fig. 1). Growth rates of newly formed branches of single 2nd (pseudo-)compartments was similar to the growth speed of branches that had only been dissected in the apical compartment (Table 2). In the case of $R$. stolonifer, only lateral branches were formed from single 2nd compartments. These branches were observed in $17.2 \%$ of the single pseudo-2nd compartments and had a mean extension rate of $15.5 \pm 14.4 \mu \mathrm{m} \mathrm{h}^{-1}$ (Table 2). This growth speed was about threefold lower than that of newly formed branches of hyphae that had only been dissected at the apex. Together, growth rate of single second compartments is lower than that of the apical compartment (Tables 1, 2). Thus, the second compartment is not self-sustaining in reaching the maximal hyphal growth rate as found at the hyphal apex.

Predictors for continued growth of dissected hyphae

Residual cytoplasmic volume of the apical compartment after dissection is a predictor of continued growth of $A$. niger after dissecting the second compartment (Tegelaar and Wösten 2017). Here, it was assessed whether growth rate, volume of the (pseudoapical compartment and/or the amount of cytoplasmic loss are predictors of continued growth of hyphal apices after dissection of $P$. chrysogenum and $R$. stolonifer in the second compartment. S. commune was not included since only $4 \%$ of its hyphae stopped growing after dissection of the 2 nd compartment (see above) hampering statistical analysis of potential predictors of continued growth. Growth rate of $P$. chrysogenum and $R$. stolonifer hyphae was distributed unimodally (Fig. 2). Relative slow- or fast-growth within these unimodal distributions was not causally linked to continued growth after dissection of the second compartment of these fungi (Data not shown). The initial volume of $P$. chrysogenum apical compartments that continued or stopped growing was $2.6 \pm 0.2$ or $2.0 \pm 0.3$ picoliter (Table 3 ). This and the fact that absolute residual cytoplasmic volume in the apical compartment after dissecting the 2nd compartment was larger for continuing apical compartments (Table 3) shows that volume of the first compartment is a predictor of continued growth in the case of $P$. chrysogenum. Differences in absolute or relative cytoplasmic volumes of pseudo-apical compartments before or after laser dissection of the pseudo-2nd compartment of $R$. stolonifer were not found between hyphae that stopped or continued their growth. The same was found for single (pseudo-)2nd compartments of $P$. chrysogenum and $R$. stolonifer and also for A. niger (Table 3). Together, these results show that absolute cytoplasmic volume is a predictor of continued growth of apical compartments of $P$. chrysogenum, while in the case of $A$. niger the relative residual cytoplasmic volume after dissection is causally linked with continued growth (Tegelaar and Wösten 2017).

\section{Discussion}

Fungal hyphae have been reported to require a minimal length or minimum number of compartments to maintain their growth rate (Trinci 1971). For instance, the peripheral growth zones of A. niger and $P$. chrysogenum would consist of 11 and 13 intact compartments and $8660 \mu \mathrm{m}$ for the non-septate hyphae of $R$. stolonifer. However, it was shown that the apical compartment of A. niger can maintain its own growth rate (Tegelaar and Wösten 2017). Thus, sub-apical compartments are not needed to support growth of the apical compartments of A. niger hyphae but were rather shown to function as back-up to maintain peripheral growth in the case of apical injury. Here, it was shown that (pseudo-)sub-apical compartments are also not needed to support growth of (pseudo-)apical compartments of the basidiomycete $S$. commune, the ascomycete $P$. chrysogenum and the mucoromycete $R$. stolonifer. Like A. niger, these subapical compartments function as a back-up system in the case of apical injury. In addition, it is shown that in 
Fig. 1 Single second compartments of $P$. chrysogenum $(\mathbf{A}, \mathbf{B})$ and $A$. niger $(\mathbf{C}, \mathbf{D}) 60(\mathbf{A}, \mathbf{C})$ and $240($ B, D) min after laser dissection. Arrows denote the location of dissection, $s$ the location of the septa and $a$ and $b$ the location of the apex of hyphae formed through the apical of basal septum, respectively
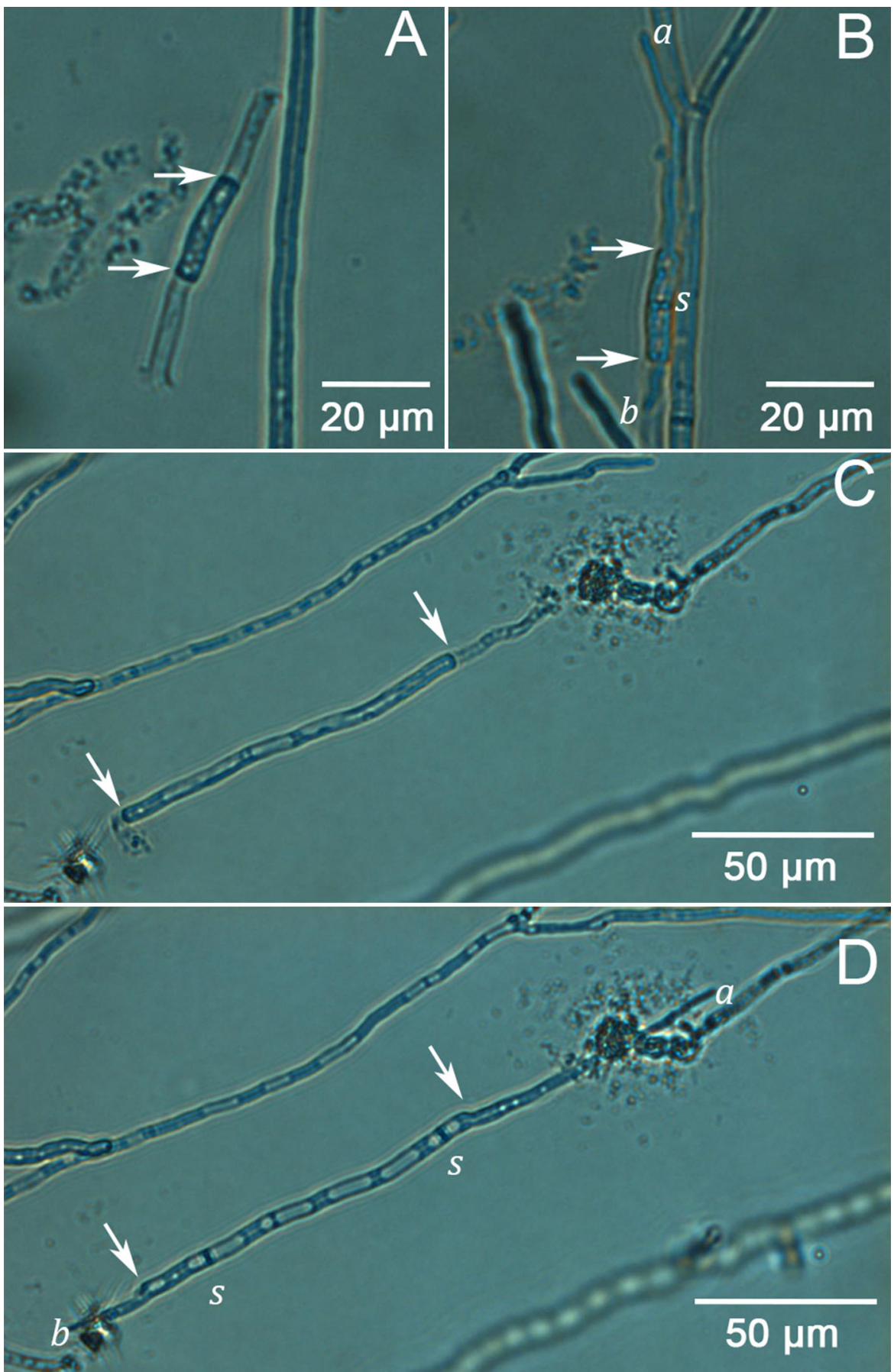

contrast to the apical compartment, the second compartment is not self-sustaining to reach the maximal growth rate of the (pseudo-)apical compartment.

A total of 9,4 , and $40 \%$ of the (pseudo-)apical compartments of $P$. chrysogenum, $S$. commune and $R$. stolonifer stopped growing after dissection, respectively. The $P$. chrysogenum hyphae that continued growing after dissection of the second compartment lost only $1 \%$ of their cytoplasm, while losses of 13 and $27 \%$ were found for $S$. commune and $R$. stolonifer, respectively. Moreover, in agreement with Tegelaar and Wösten (2017) a closed state of the 


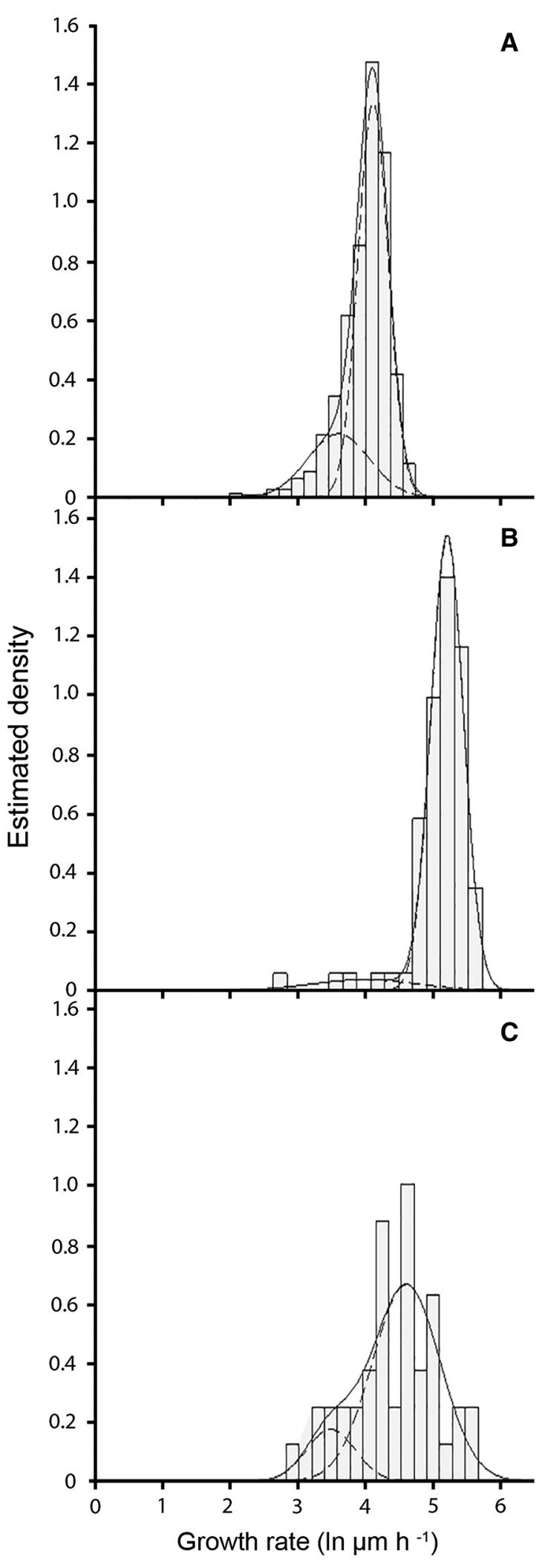

४Fig. 2 Distribution of growth rate of peripheral leading hyphae of P. chrysogenum (A), S. commune (B) and R. stolonifer (C)

apical septum in P. chrysogenum and S. commune was found not to be a significant contributor to the successful continuation of apical growth after dissection of the second compartment. In fact, $R$. stolonifer does not have septa in its vegetative hyphae but those hyphae that continued growth after dissection still maintained their initial growth rate.

The property of single (pseudo-)apical compartments to continue their growth raised the question if (pseudo-)2nd compartments also have this potential. This was assessed by cutting in both the first and third compartment or, in the case of $R$. stolonifer by cutting 500 and $1000 \mu \mathrm{m}$ from the apex. Growth from single (pseudo-)2nd compartments took place from existing or newly formed branches. The branches were formed laterally, or grew through the apical or basal septum. Apical growth of the branches of the 2nd single (pseudo-)compartments was about fourfold (R. stolonifer), 13-fold ( $P$. chrysogenum), 11-fold (A. niger; Tegelaar and Wösten 2017) and tenfold (S. commune) lower when compared to single (pseudo-)apical compartments. This lower growth rate may be caused by decreased turgor pressure (Lew 2011) or loss of $\mathrm{Ca}^{2+}$ potential (Lew 1999), secretory vesicles (Takeshita et al. 2017) or proteins (Lichius et al. 2012). In addition, the medium surrounding the detached (pseudo-)2nd compartments may be reduced in nutrients when compared to the medium the apical compartments are exposed to.

The fact that pseudo-apical and even single pseudosecond compartments of $R$. stolonifer can continue growing after being isolated from the rest of the mycelium is remarkable. Intuitively, one would assume that the pseudo-apical and pseudo-second compartments would lose their cytoplasm after dissection. However, total loss of cytoplasm occurs with low incidence, if at all. This may be explained by the capillary force of the hyphae and by organelles like vacuoles that block the opening(s) of the dissected compartments. Clearly, the presence of septa promotes the incidence of hyphal survival after dissection. However, the finding that growth of pseudoapical septa is still observed after loss of $27 \%$ of the cytoplasm suggests that $R$. stolonifer has evolved 
Table 3 Volumes of single (pseudo-)apical or single (pseudo-)2nd compartments before and after laser dissection of (pseudo-)2nd compartments and (pseudo-)apical and (pseudo-)3rd compartments, respectively. ${ }^{\mathrm{a} D a t a}$ from Tegelaar and Wösten (2017)

Volume before

laser dissection $(\mathrm{pl})$
Volume after laser dissection $(\mathrm{pl})$

Aspergillus niger

Stopping apical compartment ${ }^{\mathrm{a}}$

$6.4 \pm 1.7$

$3.9 \pm 1.8$

$\mathrm{N}=6$

Continuing apical compartment ${ }^{\mathrm{a}}$

$9.2 \pm 1.3$

$8.1 \pm 1.4$

$\mathrm{N}=43$

Stopping second compartment

$0.9 \pm 0.3$

$0.9 \pm 0.3$

$\mathrm{N}=12$

Continuing second compartment

$0.9 \pm 0.1$

$0.9 \pm 0.1$

$\mathrm{N}=62$

Penicillium chrysogenum

Stopping apical compartment

$$
2 \pm 0.3
$$

$1.8 \pm 0.4$

$\mathrm{N}=31$

Continuing apical compartment

$2.6 \pm 0.2$

$2.5 \pm 0.2$

$\mathrm{N}=53$

Stopping second compartment

$0.6 \pm 0.1$

$0.6 \pm 0.1$

$\mathrm{N}=17$

Continuing second compartment

$0.6 \pm 0.2$

$0.6 \pm 0.2$

$\mathrm{N}=40$

Rhizopus stolonifer

Stopping pseudo-apical compartment

$6.1 \pm 2.4$

$2 \pm 2.8$

$\mathrm{N}=11$

Continuing pseudo-apical compartment

$4.8 \pm 1.1$

$3 \pm 0.8$

$\mathrm{N}=13$

Stopping pseudo-second compartment

$5.1 \pm 1.1$

$2.6 \pm 0.9$

$\mathrm{N}=16$

Continuing pseudo-second compartment

$6.4 \pm 3.8$

$2.2 \pm 1.7$

$\mathrm{N}=6$

mechanisms to maintain growth after loss of a relatively high percentage of cytoplasm. Such mechanisms seem to be absent in ascomycetes and basidiomycetes and would partly compensate for the absence of septa in the aseptate fungi.

Acknowledgements This work was financed by the Netherlands Organization for Scientific Research (NWO) grant 823.02.015.

Author contributions All authors contributed to the study conception and design. Material preparation, data collection and analysis were performed by Martin Tegelaar and George van der Lans. Martin Tegelaar and Han Wösten wrote the manuscript. All authors read and approved the final manuscript.

\section{Compliance with ethical standards}

Conflict of interest The authors declare not to have any conflict of interest.

Open Access This article is licensed under a Creative Commons Attribution 4.0 International License, which permits use, sharing, adaptation, distribution and reproduction in any medium or format, as long as you give appropriate credit to the original author(s) and the source, provide a link to the Creative Commons licence, and indicate if changes were made. The images or other third party material in this article are included in the article's Creative Commons licence, unless indicated otherwise in a credit line to the material. If material is not included in the article's Creative Commons licence and your intended use is not permitted by statutory regulation or exceeds the permitted use, you will need to obtain permission directly 
from the copyright holder. To view a copy of this licence, visit http://creativecommons.org/licenses/by/4.0/.

\section{References}

Bleichrodt R, van Veluw GJ, Recter B, Maruyama J, Kitamoto K, Wösten HAB (2012) Hyphal heterogeneity in Aspergillus oryzae is the result of dynamic closure of septa by Woronin bodies. Mol Microbiol 86:1334-1344

Bleichrodt R, Hulsman M, Wösten HAB, Reinders MJ (2015a) Switching from a unicellular to multicellular organization in an Aspergillus niger hypha. MBio 6:e00111

Bleichrodt R, Vinck A, Read ND, Wösten HAB (2015b) Selective transport between heterogeneous hyphal compartments via the plasma membrane lining septal walls of Aspergillus niger. Fungal Genet Biol 82:193-200

Bos CJ, Debets AJM, Swart K, Huybers A, Kobus G, Slakhorst SM (1988) Genetic analysis and the construction of master strains for assignment of genes to six linkage groups in Aspergillus niger. Curr Genet 14:437-443

Dons JJM, de Vries OMH, Wessels JGH (1979) Characterization of the genome of the basidiomycete Schizophyllum commune. Biochim Biophys Acta 563:100-112

Lew RR (1999) Comparative analysis of $\mathrm{Ca}^{2+}$ and $\mathrm{H}^{+}$flux magnitude and location along growing hyphae of Saprolegnia ferax and Neurospora crassa. Eur J Cell Biol 78:892-902

Lew RR (2005) Mass flow and pressure-driven hyphal extension in Neurospora crassa. Microbiology 151:2685-2692

Lew RR (2011) How does a hypha grow? The biophysics of pressurized growth in fungi. Nat Rev Microbiol 9:509-518

Lichius A, Yáñez-Gutiérrez ME, Read ND, Castro-Longoria E (2012) Comparative live-cell imaging analyses of SPA-2, BUD-6 and BNI-1 in Neurospora crassa reveal novel features of the filamentous fungal polarisome. PLoS ONE 7:e30372

Moore RT, McAlear JH (1962) Fine structures of mycota. Observations on septa of ascomycetes and basidiomycetes. Am J Bot 49:86-94

Ohm RA, de Jong JF, Lugones LG, Aerts A, Kothe E, Stajich JE, de Vries RP, Record E, Levasseur A, Baker SE, Bartholomew KA, Coutinho PM, Erdmann S, Fowler TJ, Gathman AC, Lombard V, Henrissat B, Knabe N, Kuees U, Lilly WW, Lindquist E, Lucas S, Magnuson JK, Piumi F, Raudaskoski M, Salamov A, Schmutz J, Schwarze FWMR, van Kuyk PA, Horton JS, Grigoriev IV, Wösten HAB (2010) Genome sequence of the model mushroom Schizophyllum commune. Nat Biotechnol 28:957-963

Shatkin AJ, Tatum EL (1959) Electron microscopy of $\mathrm{Neu}$ rospora crassa mycelia. J Biophys Biochem Cytol 6:423-426

Stauffer JF (1961) The use of ultraviolet radiation for mutations in antibiotic producing fungi. Sci Rep Ist Super Sanita $1: 472-480$

Steinberg G, Peñalva M, Riquelme M, Wösten HAB, Harris S (2017) Cell biology of hyphal growth. Microbiolspec 5: FUNK-0034-2016

Takeshita N, Evangelinos M, Zhou L, Serizawa T, SomeraFajardo RA, Lu L, Takaya N, Nienhaus GU, Fischer R (2017) Oscillations in fungal tip growth by $\mathrm{Ca}^{2+}$ pulses. Proc Natl Acad Sci USA 114:5701-5706

Tegelaar M, Wösten HAB (2017) Functional distinction of hyphal compartments. Sci Rep 7:6039

Tegelaar M, Bleichrodt RJ, Nitsche B, Ram AFJ, Wösten HAB (2019) Subpopulations of hyphae secrete proteins or resist heat stress in Aspergillus oryzae colonies. Environ Microbiol. https://doi.org/10.1111/1462-2920.14863

Trinci APJ (1971) Influence of the width of the peripheral growth zone on the radial growth rate of fungal colonies on solid media. J Gen Microbiol 67:325-344

van Peer AF, Müller WH, Boekhout T, Lugones LG, Wösten HAB (2009) Cytoplasmic continuity revisited: closure of septa of the filamentous fungus Schizophyllum commune in response to environmental conditions. PLoS ONE 4:e5977

van Peer AF, Wang F, Van Driel KGA, De Jong JF, Van Donselaar EG, Müller WH, Boekhout T, Lugones LG, Wösten HAB (2010) The septal pore cap is an organelle that functions in vegetative growth and mushroom formation of the wood-rot fungus Schizophyllum commune. Environ Microbiol 12:833-844

Vinck A, de Bekker C, Ossin A, Ohm RA, de Vries RP, Wösten HAB (2011) Heterogenic expression of genes encoding secreted proteins at the periphery of Aspergillus niger colonies. Environ Microbiol 13:216-225

Vishniac W, Santer M (1957) The thiobacilli. Bacteriol Rev 21:195-213

Publisher's Note Springer Nature remains neutral with regard to jurisdictional claims in published maps and institutional affiliations. 